DOI: https://doi.org/10.46296/gt.v5i9edespmar.0060

\title{
SARS - COV - 2 Y TELETRABAJO: ESTILOS DE AFRONTAMIENTO FRENTE AL ESTRÉS LABORAL EN DOCENTES UNIVERSITARIOS
}

\section{SARS - COV - 2 AND TELEWORKING: COPING STYLES AGAINST WORK STRESS IN UNIVERSITY TEACHERS}

\author{
Ponce-Alencastro Jhon Alexander ${ }^{1}$; Zambrano-Saltos Roberth Olmedo ${ }^{2}$; \\ Rodríguez-Álava Leonor Alexandra ${ }^{3}$ \\ ${ }^{1}$ Estudiante de la Maestría Académica con Trayectoria de Investigación en Psicología, Mención \\ Psicoterapia. Instituto de Posgrado de la Universidad Técnica de Manabí. Portoviejo, Ecuador. \\ Correo: jhon.ponce@utm.edu.ec. ORCID ID: https://orcid.org/0000-0002-3666-7865. \\ ${ }^{2}$ Docente de la Maestría Académica con Trayectoria de Investigación en Psicología, Mención \\ Psicoterapia. Instituto de Posgrado de la Universidad Técnica de Manabí. Portoviejo, Ecuador. \\ Correo: rzambranosantos@yahoo.es. ORCID ID: https://orcid.org/0000-0002-4072-4738. \\ ${ }^{3}$ Docente de la Maestría Académica con Trayectoria de Investigación en Psicología, Mención \\ Psicoterapia. Instituto de Posgrado de la Universidad Técnica de Manabí. Portoviejo, Ecuador. \\ Correo: Irodríguez@utm.edu.ec. ORCID ID: https://orcid.org/0000-0002-3034-1311.
}

\begin{abstract}
Resumen
El estrés laboral es un problema que afecta a todas las profesiones, sobre todo durante la actual pandemia por Sars - Cov -2, se ha incrementado la sobrecarga de actividades laborales en los docentes universitarios que continúan sus actividades en la modalidad de teletrabajo. El propósito del presente trabajo fue identificar los estilos de afrontamiento que más adoptan los docentes universitarios frente al estrés laboral. Mediante un diseño descriptivo no experimental de tipo transversal en una población de 89 docentes a tiempo completo de la Facultad de Ciencias Administrativas y Económicas de la Universidad Técnica de Manabí a quienes se les aplico el cuestionario de afrontamiento al estrés (CAE). El resultado de este trabajo ha arrojado datos relevantes y pertinentes estableciéndose como estilos de afrontamiento más utilizados por los docentes: la búsqueda de apoyo social, la evitación, la expresión emocional abierta y la reevaluación positiva. Conclusión: El personal docente universitario en modalidad de teletrabajo por la emergencia sanitaria de Sars - Cov - 2 presenta estilos de afrontamiento inadecuados ante el estrés laboral que resultan poco saludables en el desarrollo de estrategias desadaptativas que disminuyen la interacción con sus pares y la capacidad de resolver problemas relacionados con sus actividades laborales.
\end{abstract}

Palabras clave: Estrés laboral, Afrontamiento, Pandemia, Docencia, Teletrabajo.

\begin{abstract}
Work stress is a problem that affects all professions, especially during the current pandemic by Sars - Cov - 2, the overload of work activities has increased in university teachers who continue their activities in the teleworking modality. The purpose of this study was to identify the coping styles that university teachers adopt the most in the face of work stress. Through a descriptive non-experimental cross-sectional design in a population of 89 full-time teachers from the Faculty of Administrative and Economic Sciences of the Technical University of Manabí, to whom the stress coping questionnaire (CAE) was applied. The result of this work has yielded relevant and pertinent data, establishing as coping styles most used by teachers: the search for social support, avoidance, open emotional expression and positive reappraisal. Conclusion: The university teaching staff in teleworking mode due to the Sars - Cov - 2 health emergency presents Información del manuscrito:
\end{abstract}

Fecha de recepción: 06 de enero de 2022.

Fecha de aceptación: 24 de febrero de 2022.

Fecha de publicación: 04 de marzo de 2022. 
inadequate coping styles in the face of work stress that are unhealthy in the development of maladaptive strategies that decrease interaction with their peers and the ability to solve problems related to their work activities.

Keywords: Work stress, Coping, Pandemic, Teaching, Telecommuting.

\section{INTRODUCCIÓN}

La emergencia sanitaria empezó con un brote de casos de neumonía en diciembre del 2019, en Wuhan (Hubei, China), causada por un nuevo tipo de coronavirus, (SARSCoV-2, anteriormente conocido como 2019-nCoV), siendo los coronavirus una familia de virus que normalmente afectan sólo a animales, aunque algunos tienen la capacidad de transmitirse a las personas. Esta enfermedad por coronavirus se llegó a extender por toda China y recibió atención mundial debido a su rápido crecimiento y cantidad de víctimas mortales (Ozamiz et al., 2020).

Es así que a partir del 11 de marzo de 2020, la Organización Mundial de la Salud (OMS) declaró al COVID-19 una pandemia, estableciéndose el periodo de alarma, restringiéndose la circulación, debiéndose realizar individualmente y limitada a actividades de primera necesidad o desplazamientos a los lugares de trabajo, reduciéndose la oferta del transporte de viajeros, cerrándose los locales de actividades culturales, artísticas, deportivas y similares; priorizándose el trabajo a distancia y suspendiendo las actividades escolares presenciales en favor de actividades educativas en línea (Balluerka Lasa et al., 2020).

En este sentido la suspensión de las clases presenciales en todos los niveles, ha dado origen a campos de acción principales como el despliegue de modalidades de aprendizaje a distancia, mediante la utilización de una diversidad de formatos y plataformas (con o sin uso de tecnología), el apoyo y movilización del personal, y las comunidades educativas en materia de atención a la salud y el bienestar integral de la comunidad estudiantil (CEPAL, 2020).

Estos cambios en la organización del trabajo han incrementado el estrés de los docentes en Europa y Latinoamérica, obedeciendo a la 
adaptación a las tecnologías de la informática y la comunicación (TIC), que se suma al aumento de labores dentro del hogar, el aislamiento y el temor al contagio del coronavirus (Robinet y Pérez, 2020).

Es así que la pandemia de la COVID - 19 ha sido percibida como una amenaza real a la vida, activando todos nuestros medios existenciales (Ponce et al., 2021) tratando de lidiar la incertidumbre ignorando las reacciones psicofísicas del estrés como una abrumadora amenaza a la salud mental.

De tal forma que al referirnos al estrés laboral, se lo define como el efecto de la presencia de factores de riesgo psicosocial, con una elevada posibilidad de causar severos efectos en la salud del personal docente $y$ en el funcionamiento organizacional de la institución educativa (Osorio y Niño, 2017).

En esta línea del pensamiento para Rodríguez et al. (2017) el estrés laboral de docentes ha sido ampliamente estudiado a nivel internacional por numerosos investigadores como Cox (1978), Lazarus y Folkman (1984) y Mc
Grath (1970), estudiándose también las estrategias de afrontamiento principalmente con relación al estrés. Por lo que en la situación pandémica actual es un tema que ha tomado mayor interés no tanto por su presencia agregada, sino por el déficit motivacional, que favorece la aparición de expectativas negativas con relación a las capacidades que tengan los docentes y la sensación de ser poco competentes, influyendo en el desarrollo personal y proyecto de vida (Cháidez y Barraza, 2018).

Teniendo especial interés las diversas variables que puedan influir en la presencia de este tipo particular de estrés en docentes, siendo una de estas, los estilos de afrontamiento que puedan adoptar para lidiar con el conflicto de cumplir sus labores a pesar de que estén diezmados los recursos de poder afrontar sin llegar al enfrentamiento ofensivo de las altas exigencias.

Este nivel de estrés se ve favorecido por el clima laboral poco favorable, impidiendo que las acciones se desarrollen de manera satisfactoria, ya que la comunicación y participación son afectadas por la estructura piramidal de las 
organizaciones, generando entre otros síntomas ansiedad, depresión apatía y resistencia al cambio (Martínez et al., 2019).

Pero todo esto ha tenido una mayor sobrecarga durante el contexto de la emergencia sanitaria ocasionada por Sars - Cov - 2 en la modalidad del trabajo docente, que de la actividad presencial tuvieron que adoptar de manera impositiva la virtualidad.

Precisando profundizar el conocimiento de aquellos procesos de regulación y afrontamiento vinculados con diversos factores intervinientes, que puedan proveer 0 limitar estrategias de intervención para menguar 0 aumentar la respuesta ante el estrés con una serie de trastornos fisiológicos y psicológicos.

A partir de los puntos planteados anteriormente surge el interés de esta investigación para responder a la pregunta, ¿Qué estilos de afrontamiento son los que más adoptan los docentes universitarios frente al estrés laboral?

Esta pregunta se despliega en el transcurso de la discusión de los resultados de este estudio, llevando a plantear como objetivo la identificación de los principales estilos de afrontamiento que más adoptan los docentes frente al estrés laboral, sus modelos explicativos y variables asociadas.

\section{METODOLOGÍA}

La investigación fue de tipo no experimental, descriptiva, sincrónica de corte transversal bajo el planteamiento metodológico del enfoque cuantitativo. Como método empírico se utilizó el cuestionario de afrontamiento al estrés (CAE) (Sandín y Chorot, 2003) para generar datos, mediante la aplicación de google forms para el desarrollo y aplicación del cuestionario.

\section{Materiales y métodos}

La población fue de 89 docentes de la Facultad de Ciencias Administrativas y Económicas de la Universidad Técnica de Manabí según el reporte de los coordinadores departamentales que cumplían con características similares entre ellas como el ser docentes a tiempo completo de la universidad, excluyéndose a los docentes a medio tiempo, aquellos que ya no eran docentes $y$ 
eliminándose aquellos que hubieran fallecido y a quienes no firman el consentimiento informado. Como instrumento se utilizó el Cuestionario de Afrontamiento del Estrés (CAE) que es una medida de autoinforme diseñada para evaluar siete estilos básicos de afrontamiento: focalizado en la solución del problema (FSP), autofocalización negativa (AFN), reevaluación positiva (REP), expresión emocional abierta (EEA), evitación (EVT), búsqueda de apoyo social (BAS), y religión (RLG). Los participantes firmaron consentimiento informado y una vez que se obtuvo los resultados del cuestionario respectivamente contestado, se procedió al diseño de una base de datos para realizar el análisis estadístico, empleándose el paquete estadístico SPSS versión 26. Se declara que no existen conflictos de intereses y se mantendrá la confidencialidad de la información.

\section{RESULTADOS}

De la población total de docentes predomino el sexo femenino en un $57 \%$ en comparación con el sexo masculino que fue del $43 \%$. Tabla 1 .

Destacando como una de las principales características generales de esta población la mayor cantidad de mujeres que forman parte del cuadro docente universitario, pudiendo dejar ver posibles diferencias en la forma como se comportan las variables del estudio en ambos sexos.

Tabla 1. Sexo de docentes universitarios.

\begin{tabular}{|c|c|c|c|c|c|}
\hline & & Frecuencia & Porcentaje & $\begin{array}{c}\text { Porcentaje } \\
\text { válido }\end{array}$ & $\begin{array}{l}\text { Porcentaje } \\
\text { acumulado }\end{array}$ \\
\hline \multirow[t]{3}{*}{ Válidos } & $\mathrm{F}$ & 51 & 57,3 & 57,3 & 57,3 \\
\hline & M & 38 & 42,7 & 42,7 & 100,0 \\
\hline & Total & 89 & 100,0 & 100,0 & \\
\hline
\end{tabular}

El grupo etario que predominó fue el de 41 a 50 años con el 50.26\%, seguido del $22.47 \%$ para el grupo de 30 a 40 años y el $21.36 \%$ para el grupo de mayores de 50 años. Tabla
2. A partir de estos resultados se observa que el desarrollo de la práctica docente va consolidándose a mayor edad, tomando como punto de mayor referencia en esta 
población el grupo de mediana edad con una considerable experiencia en el desempeño de las funciones docentes que han experimentado cambios de modalidad en la pandemia actual.

Tabla 2. Grupos etarios de los docentes universitarios

\begin{tabular}{|c|c|c|c|c|c|}
\hline & & Frecuencia & Porcentaje & $\begin{array}{c}\text { Porcentaje } \\
\text { válido }\end{array}$ & $\begin{array}{l}\text { Porcentaje } \\
\text { acumulado }\end{array}$ \\
\hline \multirow[t]{4}{*}{ Válidos } & $30-40$ & 19 & 21,3 & 21,3 & 21,3 \\
\hline & $41-50$ & 44 & 49,4 & 49,4 & 70,8 \\
\hline & $>51$ & 26 & 29,2 & 29,2 & 100,0 \\
\hline & Total & 89 & 100,0 & 100,0 & \\
\hline
\end{tabular}

Los años de antigüedad que predominaron en esta población fueron aquellos entre los 11 a 20 años con el $50.56 \%$, seguido de los que tienen más de 21 años en un $26.96 \%$ y los de 5 a 10 años de antigüedad corresponden al $22.47 \%$.

Tabla 3
Esta antigüedad laboral junto a la mayor edad guarda cierta influencia con la considerable experiencia en el desempeño de las funciones docentes.

Tabla 3. Años de antigüedad laboral.

\begin{tabular}{|c|c|c|c|c|c|}
\hline & & Frecuencia & Porcentaje & $\begin{array}{l}\text { Porcentaje } \\
\text { válido }\end{array}$ & $\begin{array}{l}\text { Porcentaje } \\
\text { acumulado }\end{array}$ \\
\hline \multirow{4}{*}{ Válidos } & $5-10$ & 49 & 55,1 & 55,1 & 55,1 \\
\hline & $11-20$ & 33 & 37,1 & 37,1 & 92,1 \\
\hline & $>21$ & 7 & 7,9 & 7,9 & 100,0 \\
\hline & Total & 89 & 100,0 & 100,0 & \\
\hline \multicolumn{6}{|c|}{$\begin{array}{l}\text { Fuente: docentes encuestados } \\
\text { Autor: Jhon Ponce Alencastro }\end{array}$} \\
\hline
\end{tabular}

Con respecto a los estilos de afrontamiento que utilizan los docentes universitarios se obtuvo que el $27 \%$ utilizan la Búsqueda de apoyo social (BAS), Evitación (EVT) en el $22.5 \%$, la Expresión emocional abierta (EEA) en el 18\%, la
Reevaluación positiva (REP) en el $11.2 \%$, la Religión (RLG) en el 9\%, la Autofocalización negativa (AFN) en el $6.7 \%$ y el Focalizado en la solución del problema (FSP) en el 5.6\%. Tabla 4 y Gráfico 1. 
Tabla 4. Estilos de afrontamiento adoptados por los docentes universitarios.

\begin{tabular}{|c|c|c|c|c|c|}
\hline & & Frecuencia & Porcentaje & $\begin{array}{c}\text { Porcentaje } \\
\text { válido }\end{array}$ & $\begin{array}{l}\text { Porcentaje } \\
\text { acumulado }\end{array}$ \\
\hline \multirow[t]{8}{*}{ Válidos } & \multirow{2}{*}{$\begin{array}{l}\text { FSP } \\
\text { AFN }\end{array}$} & 5 & 5,6 & 5,6 & 5,6 \\
\hline & & 6 & 6,7 & 6,7 & 12,4 \\
\hline & REP & 10 & 11,2 & 11,2 & 23,6 \\
\hline & EEA & 16 & 18,0 & 18,0 & 41,6 \\
\hline & EVT & 20 & 22,5 & 22,5 & 64,0 \\
\hline & BAS & 24 & 27,0 & 27,0 & 91,0 \\
\hline & RLG & 8 & 9,0 & 9,0 & 100,0 \\
\hline & Total & 89 & 100,0 & 100,0 & \\
\hline $\begin{array}{l}\text { Fuente } \\
\text { Autor: }\end{array}$ & $\begin{array}{l}\text { oce } \\
\text { on } 1\end{array}$ & $\begin{array}{l}\text { encuestac } \\
\text { ce Alencas }\end{array}$ & & & \\
\hline
\end{tabular}

Gráfico 1. Estilos de afrontamiento adoptados por los docentes universitarios.

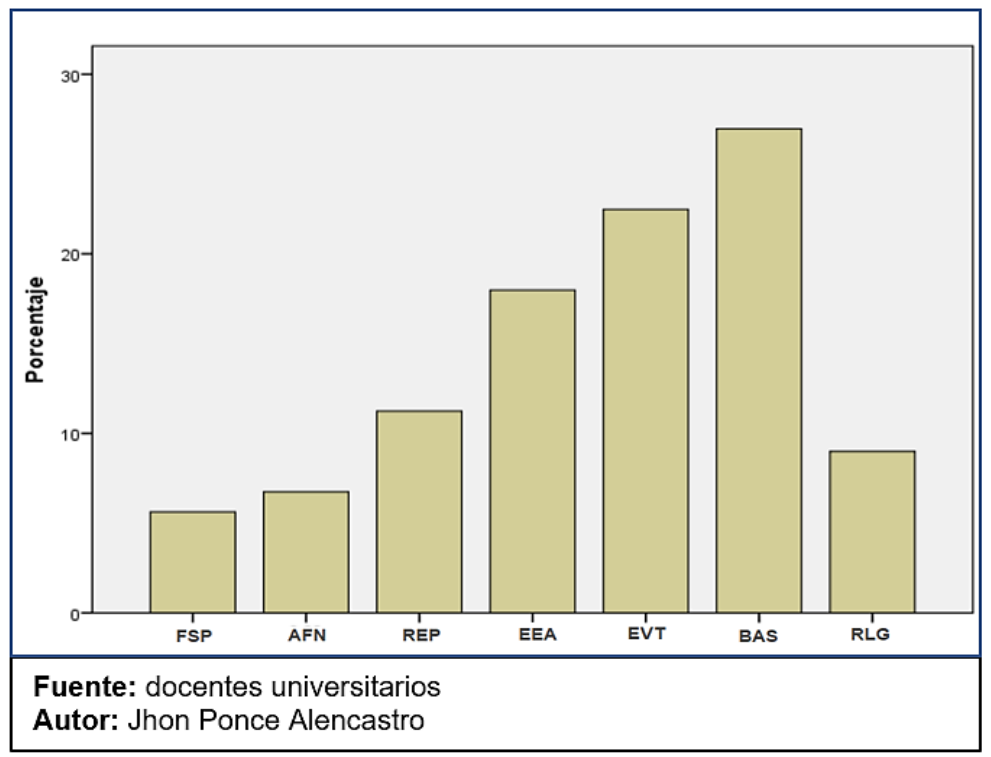

Estos resultados indicaron que la BAS fue el estilo de afrontamiento más empleado por los docentes, los cuales buscan información, ayuda y consejos de la familia y amistades que le permitan encontrar la mejor manera de resolver el problema; seguido a este la EVT fue el segundo estilo más utilizado, de manera que estos docentes no harán ni un mínimo esfuerzo por solucionar la situación que les causa malestares, lo que con lleva a empeorar su situación al pensar que el problema no tiene solución, enfocándose en otras actividades que pueden ser de su agrado para olvidarlo.

El tercer estilo más utilizado fue la EEA, teniendo manifestaciones expresivas frecuentes, descargando su ira con otras personas, comportándose en forma 
desagradable con los demás, siendo posiblemente el grupo familiar quien reciban más estas manifestaciones negativas; y como cuarto estilo más utilizado está la REP, mediante la cual los docentes se enfocan en establecer los puntos positivos de la situación problemática, pensando que a pesar de estar atravesando una desagradable situación siempre existe una gran enseñanza de vida, lo cual también los puede llevar a un estado de resignación en base a una esperanza idealizada.

Entre los estilos de afrontamiento al estrés menos utilizados tenemos a la $R L G$, en la que los docentes tratan de buscar apoyo a base de su fe en seres espirituales para solucionar su situación problemática asistiendo a la iglesia y rezando para que todo mejore; seguido está la AFN que mantendrá la situación problemática a pesar de que se intente evitar, lo que nos muestra que en los docentes siempre existirá cierto nivel de estrés, que podría condicionar un nivel moderado del mismo. Negando la situación problemática, valorándola en forma distorsionada, culpabilizándose de lo que pasa sintiéndose incapaz de encontrar una solución.
$Y$ finalmente el FSP que demuestra la poca capacidad de ciertos docentes para tomar decisiones rápidas y claras ante la situación problemática, sin la posibilidad de evaluar las causas y razones de esta situación, sin que se pueda concretar un plan que ayude a enfrentar o solucionar el problema actual.

\section{DISCUSIÓN}

Esta investigación permitió explorar los estilos de afrontamiento en una determinada población de docentes universitarios en situación de teletrabajo en el contexto de la pandemia por Sars - Cov -2 .

El sexo con mayor predominio en la población encuestada fue el femenino, este resultado fue similar a la investigación de Aguilar Acosta y Mayorga Lascano (2020) en Ecuador con una población de 91 docentes de los cuales el 58,2\% correspondió al sexo femenino y el $41,8 \%$ al sexo masculino.

Lo mismo que la investigación de Roldán Bazurto y Díaz Macías (2021) en Ecuador con una población conformada por 31 docentes, de los cuales el $58 \%$ de la muestra estudiada correspondió al 
género femenino y el $42 \%$ restante son de género masculino

En referencia a la edad de los docentes encuestados, el grupo etario poblacional de 41 a 50 años tuvo el más alto porcentaje, seguido por el grupo de 30 a 40 años y el grupo de mayores de 50 años.

Estos resultados son similares a los encontrados en una investigación con 112 docentes universitarios de Ecuador llevada a cabo por Paredes et al. (2021), en los que el grupo etario comprendido de 36 a 55 años constituyo el $76 \%$, siendo el más alto en aquel estudio.

Así mismo en el estudio realizado por Calderón Delgado (2020) en Perú en base a una muestra de 300 docentes, predomino el grupo de 36 a 65 años constituyendo el $75 \%$ del total de esta población.

La edad predominante de la población de docentes universitarios de la presente investigación está en concordancia con lo referido por Oramas Viera et al. (2007) que después de los 40 años, aparecen toda una serie de acontecimientos en la vida del sujeto que dejan su impronta en la personalidad, haciéndose frecuentes reflexiones sobre el proyecto de vida y los logros que se han tenido, constituyendo elementos importantes que convocan a la persona a centrarse en sí misma, buscando fuentes de autorrealización que pueden ser muy diversas, siendo el trabajo una de ellas.

En la investigación llevada a cabo en los docentes de la Universidad Técnica de Manabí la antigüedad laboral en el desempeño docente en mayor medida correspondió al grupo de 11 a 20 años con el 50, 5\%, los que tienen más de 21 años en un $26,9 \%$ y finalmente los de 5 a 10 años en un $22,4 \%$.

De acuerdo con una investigación realizada en México por Escudero Macluff et al. (2013), destacan los profesores que llevan 13 años trabajando, siendo el que mayor porcentaje y el resto de la población encuestada tiene más de 20 años de servicio laboral docente. Lo que concuerda de cierta manera con los datos de la presente investigación con respecto a la antigüedad laboral.

La población que participó en esta investigación debió responder el Cuestionario de Afrontamiento del Estrés (CAE) de Bonifacio Sandín y 
Paloma Chorot (2003) validado para la población latinoamericana, observándose que los docentes utilizan principalmente la BAS en un $26 \%$. El mismo que de acuerdo con Sandín y Chorot (2003) consiste en poder contar los sentimientos a familiares o amigos, pedir consejo o información a parientes o amigos, hablar con amigos o parientes para ser tranquilizado, pedir orientación sobre el mejor camino a seguir y expresar los sentimientos a familiares o amigos.

Esto concuerda con la investigación llevada a cabo en Chile por García et al. (2016) en una población de 194 docentes destacando el BAS como un mediador total entre la aceptación y el bienestar psicológico, constituyendo uno de los principales estilos de afrontamiento ante el estrés empleados.

A pesar de lo referido por Sánchez Álvarez (2009) que ante la presencia de estrés los problemas interpersonales se reflejaran en la comunicación como un problema en los vínculos con la familia y el entorno. Por lo que la BAS se convertiría en una de las últimas estrategias a considerar por la creencia de que no se contará con el apoyo suficiente que él requiere para solucionar un problema (Cacay Ramos, 2021).

En la investigación realizada en los docentes de la Universidad Técnica de Manabí, no se ha presentado de esta manera, porque mantienen la comunicación con sus vínculos más cercanos como la familia y las amistades, favoreciendo la solución de conflictos, puesto que se tendrá mayor predisposición en acudir a estas personas para solicitar apoyo con la seguridad que se lo ofrecerán sin ningún problema (Cacay Ramos, 2021) influyendo de esta manera la dinámica familiar en el bienestar psicológico de la persona en la búsqueda de soluciones, pero puede cambiar a lo largo del tiempo según las circunstancias y experiencias a las que se deba enfrentar (Martínez et al., 2017). Por eso este estilo de afrontamiento constituye el más utilizado por esta población.

Este estilo es un indicador positivo que se asocia al esfuerzo, trabajo personal, resolución de problemas y manejo de las emociones para reducir los momentos de tensión (Marsollier y Expósito, 2021b) que justificaría porque el estrés laboral no ha llegado a niveles severamente 
altos, pero nos deja abierta otra línea de estudio sobre la funcionalidad de las familias de estos docentes en la respuesta cognitiva, conductual y emocional ante la carga de estrés vincular a lo largo del tiempo mientras sigan presentes las fuentes estresoras laborales.

El EVT es el segundo más empleado por los docentes de la Universidad Técnica de Manabí en un $22 \%$, el mismo que de acuerdo con (Piergiovanni y Depaula, 2018) se caracteriza porque la persona se concentra en otras cosas y prefiere no pensar en el problema, tratándose de una variable de huida - de escape de la situación estresante.

Este resultado concuerda con el encontrado en una investigación realizada en Argentina por (Marsollier y Expósito, 2021b) en una población de 777 docentes quienes prefieren la EVT como uno de los principales estilos de afrontamiento al estrés laboral.

Que es recíproco con los datos provenientes de la investigación de Paredes et al. (2021) en una población de 335 docentes universitarios en Ecuador siendo el EVT uno de los más utilizados.

La EEA es el tercer estilo de afrontamiento más empleado por los docentes de la Universidad Técnica de Manabí en un $18 \%$, el mismo que de acuerdo con Sandín y Chorot (2003) se caracteriza por descargar el mal humor con los demás, insultar a otras personas, comportarse hostilmente, agredir e irritarse con alguien, luchar y desahogarse emocionalmente.

Guardando relación con los resultados de la investigación por Supo Supo (2020) en 115 docentes de Perú, donde el EEA es uno de los más utilizados relacionándose de forma significativa con un nivel de intensidad medio y de tipo inversa con otros estilos de afrontamiento.

Similares resultados se encontraron en el estudio por Acosta y Greca (2020) en una población de 50 docentes en Argentina, predominando el EEA entre uno de los más empleados frente al estrés laboral.

La REP es el cuarto estilo de afrontamiento más empleado por los docentes de la Universidad Técnica de Manabí en un $11.2 \%$. De acuerdo 
con Gantiva et al. (2010) a través de esta estrategia se busca aprender de las dificultades, identificando los aspectos positivos del problema, esto contribuye a tolerar la problemática y a generar pensamientos positivos que favorecen enfrentar la situación.

Estos resultados son similares con el estudio realizado en México por Carmona García et al. (2015) en 263 docentes, donde el REP fue uno de los principales estilos de afrontamiento empleados ante el estrés.

Los estilos de afrontamiento al estrés menos empleados fueron RLG $9 \%$, AFN $6.7 \%$ y FSP $5.6 \%$.

Estos datos son similares a los reportados por una investigación realizada en Colombia por Muñoz et al. (2020) con 274 docentes el estilo RLG fue uno de los utilizados en menor proporción, así mismo el estilo AFN fue uno de los menos empleados en una investigación realizada en España por Vicente y Gabari (2019) en una población de 67 docentes. $Y$ finalmente el estilo FSP fue el menos utilizado de acuerdo a la investigación de Flor Egas (2021) en 242 docentes en Ecuador.

\section{CONCLUSIONES}

Este estudio demostró que los estilos de afrontamiento más utilizados son el BAS, EVT, EEA, REP y los menos utilizados son RLG, AFN, FSP por los docentes de la Facultad de Ciencias Administrativas y Económicas de la Universidad Técnica de Manabí a tiempo completo en modalidad de teletrabajo por la emergencia sanitaria debido a la pandemia de Sars - Cov -2 .

De manera que estos estilos de afrontamiento ante el estrés laboral son el producto de la interacción de varios factores estresores que generan diferentes tipos de respuestas adaptativas que en la población del presente estudio han estado de cierta forma influenciadas por la manera en la que se adoptó la modalidad del teletrabajo.

De los 89 docentes universitarios predominó el sexo femenino con edades entre los 41 y 50 años con 11 a 20 años de antigüedad en el ejercicio docente, destacando que se encuentran en el grupo de madurez laboral, pero también es el más afectado probablemente por la adición de las tareas de cuidados del hogar, de los hijos y el cumplimiento 
con las exigencias del teletrabajo de acuerdo con la asignación de la carga laboral.

\section{BIBLIOGRAFÍA}

Acosta, M. A., \& Greca, M. E. (2020). Estilos de afrontamiento del estrés en maestros de educación especial y maestros de enseñanza primaria, de la ciudad de Villaguay [Tesis posgrado, Universidad Católica Argentina].

https://repositorio.uca.edu.ar/ handle/123456789/10593

Aguilar Acosta, A., \& Mayorga Lascano, M. (2020). Relación entre Estrés Docente y Síndrome de Burnout en docentes de la Zona 3 de Ecuador. UNIANDES EPISTEME Revista digital de Ciencia, Tecnología e Innovación, 7(2), 265-278.

Balluerka Lasa, M. N., Gómez
Benito, J., $\begin{array}{r}\text { Hidalgo } \\ \text { Montesinos, M. }\end{array}$ D.,
Gorostiaga Manterola, M. A.,
Espada Sánchez, J. P.,
Padilla García, J. L., \& Santed
Germán, M. Á. (2020). Las
consecuencias psicológicas
de la COVID-19 y el
confinamiento. Informe de
investigación (Primera, Vol.
1). Servicio Editorial de la
Universidad del País
Vasco/Euskal
Unibertsitatearen Argitalpen

Zerbitzua.

https://addi.ehu.es/handle/10 810/45924

Cacay Ramos, M. (2021). Estrategias de afrontamiento en docentes relacionadas con el confinamiento y estrés desde la perspectiva cognitivo conductual [Tesis posgrado, Universidad Técnica de Machala].

http://repositorio.utmachala.e du.ec/bitstream/48000/16788/ 1/ECFCS-2021-PSCDE00011.pdf

Calderón Delgado, H. (2020). Estilos de afrontamiento al estrés y ansiedad en docentes de la Región San Martín, durante la pandemia COVID-19. [Posgrado, Universidad Peruana Unión]. http://200.121.226.32:8080/bi tstream/handle/20.500.12840 /3804/Havy_Trabajo_Bachille rato_2020.pdf?sequence

Carmona García, L., Vega Villanueva, N., Carrillo Vargas, E., Lozano Ramírez, D., \& Burciaga Robles, J. (2015). Estilos de afrontamiento de profesoras expuestas a violencia de género. Revista Electrónica de Psicología Iztacala, 18(4), 1747-1762.

CEPAL. (2020). La educación en tiempos de la pandemia de COVID-19. Informe COVID 19 CEPAL - UNESCO, Agosto, 21. 
Cháidez, J., \& Barraza, A. (2018). Afrontamiento al estrés y su relación con el tipo de jornada laboral en docentes de educación primaria. Informes Psicológicos, 18(2), 63-75. https://doi.org/10.18566/infpsi c.v18n2a04

Escudero Macluff, J., Ortíz García, J., \& Deffín Beltrán, L. (2013). La antigüedad en el trabajo y su relación con el síndrome de quemado por el trabajo (burnout) en los profesores del área. Ciencia Administrativa, 2, 19-25.

Flor Egas, R. (2021). Estrategia de Afrontamiento para Síntomas Primarios del Síndrome de Burnout del Sistema Educativo. Revista Universidad César Vallejo, 3(1), 16.

Gantiva, C., Luna, A., Dávila, A., \& Salgado, M. (2010). Estrategias de afrontamiento en personas con ansiedad. Psychologia, 4(1), 63-70. https://doi.org/10.21500/1900 2386.1159

García, F. E., Manquián, E., \& Rivas, G. (2016). Bienestar psicológico, estrategias de afrontamiento y apoyo social en cuidadores informales. Psicoperspectivas, 15(3), 101-111.

https://doi.org/10.5027/PSIC OPERSPECTIVAS-VOL15ISSUE3-FULLTEXT-789
Marsollier, R., \& Expósito, C. (2021). Afrontamiento docente en tiempos de COVID-19. CienciAmérica, 10(1), 35-54. https://doi.org/10.33210/ca.v1 $0 i 1.357$

Martínez, H., Rodríguez, L., \& Cobeña, K. (2019). Estrés laboral en los docentes del circuito 03 distrito $13 \mathrm{~d} 11$ de la zona 04 de educación y estresores psicosociales prevalentes. Revista Cognosis, 4(1), 83-98.

Martínez, J., Amador, B., \& Guerra, M. (2017). Estrategias de afrontamiento familiar $y$ repercusiones en la salud familiar: Una revisión de la literatura. Enfermería Global Revista electrónica trimestral de Enfermería, 16(3), 576. https://doi.org/10.6018/egloba I.16.3.255721

Muñoz, C., Correa, C., \& Matajudíos, J. (2020). Síndrome de Burnout y estrategias de afrontamiento en docentes de primera infancia. Revista Espacios, 41(37), 145-158.

Oramas Viera, A., Almirall Hernández, P., \& Fernández, I. (2007). Estrés Laboral y el Síndrome de Burnout en Docentes Venezolanos. Salud de los Trabajadores, 15(2), 71-87.

Osorio, J., \& Niño, L. (2017). Estrés laboral: Estudio de revisión. Diversitas: Perspectivas en Psicología, 13(1), 81-90. 
https://doi.org/10.15332/s 179 4-9998.2017.0001.06

Ozamiz, N., Dosil, M., Picaza, M., \& Idoiaga, N. (2020). Niveles de estrés, ansiedad y depresión en la primera fase del brote del COVID-19 en una muestra recogida en el norte de España. Cadernos de Saúde Pública, 36(4). https://doi.org/10.1590/0102$311 \times 00054020$

Paredes, M., Hermes, V., Lozada, D., Guale, J., \& Guzmán, J. (2021). Burnout y afrontamiento en docentes universitarios de Ecuador. Revista del Grupo de Investigación en Comunidad y Salud, 6(4), 39-54.

Piergiovanni, L. F., \& Depaula, P. D. (2018). Autoeficacia y estilos de afrontamiento al estrés en estudiantes universitarios. Ciencias Psicológicas, 12(1), 17-23.

https://doi.org/10.22235/cp.v1 2i1.1591

Ponce, J., Arredondo, K., \& Mera, M. (2021). Ansiedad social: Intervención desde la terapia de aceptación y compromiso a propósito de un caso. Revista Científica Arbitrada en Investigaciones de la Salud GESTAR. ISSN: 2737-6273., 4(8 Ed. esp.), 104-129. https://doi.org/10.46296/gt.v4i 8edesp.0035

Robinet, A., \& Pérez, M. (2020). Estrés en los docentes en tiempos de pandemia Covid19. Polo del Conocimiento, 5(12), 637-653-653. https://doi.org/10.23857/pc.v5 i12.2111

Rodríguez, J. A., Guevara, A., \& Viramontes, E. (2017). Síndrome de burnout en docentes. IE Revista de Investigación Educativa de la REDIECH, 8(14), 45-67. https://doi.org/10.33010/ie_rie _rediech.v8i14.39

Roldán Bazurto, M., \& Díaz Macías, T. (2021). Afrontamiento al estrés por teletrabajo en docentes de la Unidad Educativa Emilio Bowen Roggiero. Dominio de las Ciencias, 7(1), 133-146. http://dx.doi.org/10.23857/dc. v7i1.1695

Sánchez Álvarez, P. (2009). Cómo afrontar el estrés docente: Proyecto de investigación. Red de información educativa, 88.

Sandín, B., \& Chorot, P. (2003). Cuestionario de afrontamiento del estrés (CAE): Desarrollo y validación preliminar. Revista de Psicopatología y Psicología Clínica, 8(1), 3953.

https://doi.org/10.5944/rppc.v ol.8.num.1.2003.3941

Supo Supo, K. L. (2020). Afrontamiento del estrés y bienestar psicológico en docentes del distrito de LoS Olivos. Lima, 2020 [Tesis 
posgrado, Universidad César

Vallejo]. Lima norte.

https://repositorio.ucv.edu.pe/

handle/20.500.12692/54182

Vicente, M. I., \& Gabari, M. I. (2019).

Burnout y Factores de

Resiliencia en Docentes de

Educación Secundaria.

International Journal of

Sociology of Education, 8(2), 127-152.

https://doi.org/10.17583/rise.2

019.3987 